\title{
Hypertension in Pediatric Acute Lymphoblastic Leukemia Patients: Prevalence, Impact, and Management Strategies
}

\author{
Lindsey Murphy' \\ Kelly Maloney ${ }^{1,2}$ \\ Lia Gore ${ }^{1,2}$ \\ Eliza Blanchette ${ }^{3}$ \\ 'Department of Pediatrics, Sections of \\ Hematology/Oncology/Bone Marrow \\ Transplant-Cellular Therapeutics, \\ University of Colorado School of \\ Medicine and Children's Hospital \\ Colorado, Aurora, CO, USA; ${ }^{2}$ University \\ of Colorado Cancer Center, Aurora, CO, \\ USA; ${ }^{3}$ Department of Pediatrics, Section \\ of Nephrology, University of Colorado \\ School of Medicine and Children's \\ Hospital Colorado, Aurora, CO, USA
}

\begin{abstract}
Acute lymphoblastic leukemia (ALL) is the most common cancer diagnosed in children under the age of 18 . While modern diagnostic technologies, risk-stratification, and therapy intensification have led to outstanding outcomes for many children with ALL, the side effects and consequences of therapy are not to be underestimated. Hypertension is a well-known acute and chronic side effect of treatment for childhood ALL, although limited data are available regarding the prevalence of hypertension in children undergoing treatment for ALL. In this review of hypertension in pediatric ALL patients, we examine the existing data on incidence and prevalence during treatment and in pediatric ALL survivors. We describe independent risk factors for development of hypertension along with treatmentrelated causes. Long-term consequences and the risk to survivors of pediatric ALL are further defined. While many ALL patients require antihypertensive medications during some portion of their treatment, there are no clear guidelines on treating inpatient hypertension given challenges that exist in recognizing and managing hypertension in this setting and in this population. Here, we propose an algorithmic approach to diagnose and treat pediatric ALL patients with HTN, along with monitoring and continuation versus cessation of antihypertensive therapy as an outpatient.
\end{abstract}

Keywords: hypertension, pediatric, leukemia, ALL, prevalence, management

\section{Background}

Acute lymphoblastic leukemia (ALL) is the most common pediatric malignancy, accounting for almost one-third of all childhood cancers. ${ }^{1}$ Due to progress in treatment and advances in supportive care, the long-term survival rates of children with ALL have improved remarkably over the last 50 years and now approach $90 \%$. ${ }^{1-3}$ However, improved survival means an increased number of survivors who are at risk of treatment-related morbidity and mortality. It is well known that hypertension is one of the acute and chronic complications of pediatric leukemia treatment, but limited data are available regarding the prevalence of hypertension (HTN) in children undergoing treatment for pediatric ALL.

\section{Current Hypertension Guidelines}

In 2017, the American Academy of Pediatrics published an updated clinical practice guideline for the screening and management of high blood pressure in children and adolescents. This guideline had not been updated since 2004, with the most clinically relevant revision to include new normative pediatric blood pressure tables
Correspondence: Lia Gore

Tel +17207776458

Fax +17207777339

Email lia.gore@cuanschutz.edu 
based on normal weight children (excluding overweight and obese children) and including a simplified blood pressure (BP) classification for adolescents $\geq 13$ years of age that aligns with adult BP guidelines. ${ }^{4,5}$ Other changes include replacement of the term "pre-hypertension" with "elevated blood pressure" for values that are $\geq 90$ th percentile but $<95^{\text {th }}$ percentile for age and height. For children, thresholds for the definitions of elevated blood pressure and HTN according to age and height remain the same: blood pressure $\geq 90$ th and $<95$ th percentile designates elevated blood pressure and $\geq 95$ th percentile indicates HTN. For adolescents $\geq 13$ years of age, the criteria for high blood pressure now align with the updated adult guideline that defines measurements $\geq 120 / 80$ as elevated blood pressure and $\geq 130 / 80$ as meeting criteria for HTN. See Table 1 for a detailed side by side comparison of the new and old guidelines.

\section{Rates of Hypertension}

As previously stated, data on the incidence and prevalence of HTN in pediatric ALL patients are limited. In the general pediatric population, the prevalence of HTN in children and adolescents is approximately $3.5 \%$, with elevated blood pressure occurring in another 2.2 to $3.5 \%$ of children. ${ }^{4}$ For pediatric patients receiving treatment for ALL, $12-45 \%$ of patients who were normotensive before starting ALL therapy developed HTN during induction chemotherapy. ${ }^{6-10}$ In a large study of 5578 unique patients receiving induction chemotherapy, $14.7 \%$ received antiHTN medications during their initial hospital admission. ${ }^{9}$ This HTN is generally asymptomatic and typically resolves in the majority of patients by day 90 of treatment. $^{8,10}$ This wide incidence range may be related to heterogeneity in ALL treatment protocols (with highest incidence rates reported in older regimens when chemotherapy was more frequently combined with radiotherapy), inconsistent categorization of HTN, as well as the variations in patient populations among different studies. $^{11}$ Prior to 2017 and version 5.0 of the US National Cancer Institute's Common Terminology Criteria for Adverse Events (CTCAE), HTN was specified based only on adult HTN definitions which likely contributed to the inconsistent categorization as well as likely underestimated pediatric HTN Table 2. For example, recent published COG studies for standard and high-risk acute lymphoblastic leukemia report HTN in $2.8-4.6 \%$ of patients treated on these protocols. ${ }^{12,13}$

Fewer data are published regarding the prevalence of HTN at the time of presentation in patients with newly diagnosed ALL. Attard-Montalto et al described a small cohort of patients in which $27 \%$ were found to be hypertensive at the time of presentation. All reported patients were normotensive at follow up by 18 months after initiation of induction chemotherapy. ${ }^{7}$

\section{Steroid-Induced Hypertension}

One of the factors contributing to improved cure rates in pediatric ALL has been the intensive use of glucocorticoids during induction therapy and as pulse therapy during maintenance. It has been extensively documented that glucocorticoids induce HTN. The principal mechanism of corticosteroid-induced HTN is the overstimulation of the mineralocorticoid receptor. This results in sodium retention in the kidney, followed by volume expansion, and a subsequent increase in blood pressure. ${ }^{8}$ Studies have demonstrated decreased odds for developing HTN in those treated with dexamethasone, with its lesser mineralocorticoid potency, compared to prednisone. ${ }^{9,10}$ There is also evidence for altered hypothalamus-pituitary-adrenal axis functioning in systemic HTN. ${ }^{14}$ One study evaluated the genotypes for

Table I Comparison of Definitions of the Updated 2017 American Academy of Pediatrics Clinical Practice Guideline for Screening and Management of High Blood Pressure in Children and Adolescents versus the 2004 Fourth Report on the Diagnosis, Evaluation, and Treatment of High Blood Pressure in Children and Adolescents

\begin{tabular}{|l|l|l|l|}
\hline \multirow{2}{*}{} & \multicolumn{2}{|c|}{2017 AAP CPG } & \multicolumn{2}{c|}{ 2004 Fourth Report } \\
\cline { 2 - 4 } & $<13$ Years & $\geq 13$ Years & \\
\hline Normal BP & $<90$ th percentile & $<120 /<80$ & $<90$ th percentile \\
Elevated BP* & $\geq 90$ th to $<95$ th percentile or $120-129 /<80$ & $120-129 /<80$ & $\geq 90^{\text {th }}$ to $<95^{\text {th }}$ percentile or $>120 / 80$ \\
Stage I HTN & $\geq 95$ th to $<95$ th percentile $+12 \mathrm{mmHg}$ or $130 / 80$ to $139 / 89$ & $130-139 / 80-89$ & $\geq 95$ th to $<99$ th percentile $+5 \mathrm{mmHg}$ \\
Stage $2 \mathrm{HTN}$ & $\geq 95$ th percentile $+12 \mathrm{mmHg}$ or $\geq 140 / 90$ & $\geq 140 / 90$ & $\geq 99$ th percentile $+5 \mathrm{mmHg}$ \\
\hline
\end{tabular}

Notes: *Referred to as "pre-hypertension" in the 2004 Fourth Report. Data from Flynn JT, Kaelber DC, Baker-Smith CM, et al. Clinical practice guideline for screening and management of high blood pressure in children and adolescents. Pediatrics. 2017;140:3 $3^{4}$ and Falkner B, Daniels SR. Summary of the fourth report on the diagnosis, evaluation, and treatment of high blood pressure in children and adolescents. Hypertension. 2004;44(4):387-388. ${ }^{40}$ 
Table 2 Comparison of Version 4.0 and Version 5.0 of the National Cancer Institute's Common Terminology Criteria for Adverse Events (CTCAE) Grading (https://ctep.cancer.gov/protocoldevelopment/electronic_applications/Ctc.htm) ${ }^{41}$

\begin{tabular}{|c|c|c|c|c|c|}
\hline & Grade I & Grade 2 & Grade 3 & Grade 4 & Grade 5 \\
\hline $\begin{array}{l}\text { CTCAE } \\
\text { Version } \\
4.0\end{array}$ & $\begin{array}{l}\text { Prehypertension } \\
\text { (systolic BP I20- } \\
139 \mathrm{mmHg} \text { or } \\
\text { diastolic BP } 80- \\
89 \mathrm{mmHg} \text { ) }\end{array}$ & $\begin{array}{l}\text { Stage I hypertension (systolic } \\
\text { I40-159mmHg or diastolic BP } \\
90-99 \mathrm{mmHg} \text { ); medical } \\
\text { intervention indicated; } \\
\text { recurrent or persistent ( } \geq 24 \\
\text { hours); symptomatic increase } \\
\text { by }>20 \mathrm{mmHg} \text { (diastolic) or to } \\
>140 / 90 \mathrm{mmHg} \text { if previously } \\
\text { WNL; monotherapy indicated } \\
\text { Pediatric: recurrent or } \\
\text { persistent ( } \geq 24 \text { hours) BP } \\
>\text { ULN; monotherapy indicated }\end{array}$ & $\begin{array}{l}\text { Stage } 2 \text { hypertension (systolic } \\
\mathrm{BP} \geq 160 \mathrm{mmHg} \text { or diastolic BP } \\
\geq 100 \mathrm{mmHg} \text { ); medical } \\
\text { intervention indicated; more } \\
\text { than one drug or more } \\
\text { intensive therapy than } \\
\text { previously used indicated } \\
\text { Pediatric: Same as adult }\end{array}$ & $\begin{array}{l}\text { Life-threatening consequences } \\
\text { (eg, malignant hypertension, } \\
\text { transient or permanent } \\
\text { neurologic deficit, } \\
\text { hypertensive crisis); urgent } \\
\text { intervention indicated } \\
\text { Pediatric: Same as adult }\end{array}$ & Death \\
\hline $\begin{array}{l}\text { CTCAE } \\
\text { Version } \\
5.0\end{array}$ & $\begin{array}{l}\text { Adult: Systolic } \\
\text { BP I20- } \\
\text { I39mmHg or } \\
\text { diastolic BP } 80- \\
89 \mathrm{mmHg} \\
\text { Pediatric: } \\
\text { Systolic/diastolic } \\
\text { BP >90th } \\
\text { percentile but } \\
<95 \text { th percentile } \\
\text { Adolescent: BP } \\
\geq 120 / 80 \text { even if } \\
<95 \text { th percentile }\end{array}$ & $\begin{array}{l}\text { Adult: Systolic BP I40- } \\
159 \mathrm{mmHg} \text { or diastolic } 90- \\
99 \mathrm{mmHg} \text { if previously WNL; } \\
\text { change in baseline medical } \\
\text { intervention indicated; } \\
\text { recurrent or persistent ( } \geq 24 \\
\text { hours); symptomatic increase } \\
\text { b }>20 \mathrm{mmHg} \text { (diastolic) or to } \\
>140 / 90 \mathrm{mmHg} \text {; monotherapy } \\
\text { indicated initiated } \\
\text { Pediatric and adolescent: } \\
\text { recurrent and/or persistent } \\
\text { ( } \geq 24 \text { hours) BP >ULN; } \\
\text { monotherapy indicated; systolic } \\
\text { or diastolic BP between } 95 \text { th } \\
\text { percentile and } 5 \mathrm{mmHg} \text { above } \\
99 \text { th percentile } \\
\text { Adolescent: Systolic between } \\
\text { I30-I39 or diastolic between } \\
80-89 \text { even if < } 95 \text { th percentile }\end{array}$ & $\begin{array}{l}\text { Adult: Systolic BP } \geq 160 \mathrm{mmHg} \\
\text { or diastolic BP } \geq 100 \mathrm{mmHg} \\
\text { medical intervention indicated; } \\
\text { more than one drug or more } \\
\text { intensive therapy than } \\
\text { previously used indicated } \\
\text { Pediatric and adolescent: } \\
\text { Systolic and/or diastolic } \\
>5 \mathrm{mmHg} \text { above } 99 \text { th percentile }\end{array}$ & $\begin{array}{l}\text { Adult and Pediatric: Life- } \\
\text { threatening consequences (eg, } \\
\text { malignant hypertension, } \\
\text { transient or permanent } \\
\text { neurologic deficit, } \\
\text { hypertensive crisis); urgent } \\
\text { intervention indicated }\end{array}$ & Death \\
\hline
\end{tabular}

203 candidate polymorphisms in genes likely to affect the pharmacokinetics or pharmacodynamics of glucocorticoids and other anti-leukemic agents in childhood ALL patients with HTN. This study only found variants in candidate genes affecting the alteration of the hypothalamus-pituitary-adrenal axis function in hypertensive pediatric ALL patients, and not with polymorphisms associated with the mineralocorticoid receptor. ${ }^{8}$

\section{Risk Factors for the Development of Hypertension}

Certain risk factors unrelated to leukemia or its treatment are known to increase the risk of developing HTN in children. Family history is a well-defined independent risk factor for development of HTN, especially if one or both of a child's biological parents have HTN. ${ }^{15}$ Obesity is also a known risk factor for the development of HTN in children, and obese children have an approximately 3 times higher risk of HTN requiring intervention than their normal-weight counterparts (consistent with data demonstrating that obese patients receiving induction and consolidation phases of chemotherapy for ALL are 3 times more likely to require treatment for their HTN). ${ }^{3,16}$ The prevalence of elevated BP is higher among Hispanic and non-Hispanic African American children compared with non-Hispanic white children, and according to the new BP guidelines from the AAP, high BP is consistently greater in boys than in girls $(15-19 \%$ vs $7-12 \%){ }^{4}$ 
Many additional risk factors have been identified that increase the risk for development of HTN in children in the ALL population, including exposure to corticosteroids, cytotoxic drugs, and cranial radiation. ${ }^{17}$ For a certain subset of pediatric ALL patients, specifically those with ALL blasts positive for the Philadelphia $(\mathrm{PH})$ chromosome and PH-like ALL alterations, an additional drug class of tyrosine kinase inhibitors (TKIs) is added to the treatment regimen to target and inhibit the fusion protein produced by the chromosome translocation. HTN is a known side effect of most TKIs, though HTN Incidence varies for each drug in this class and is influenced by dose, preexisting conditions, and indication for TKI. ${ }^{18}$ Fortunately, in recent decades, cumulative doses of potentially cardiotoxic treatments for ALL have been reduced and cranial radiotherapy is only used very rarely in children, primarily those with very high risk features or those who relapse with central nervous system disease. ${ }^{19}$ In addition to these factors, blood transfusions, pain, and infections may lead to transient or temporary elevated blood pressure which may not require long-term management interventions. ${ }^{7}$

Additional studies have demonstrated that acute kidney injury (AKI) is an independent predictor of BP abnormalities. ${ }^{20}$ Childhood ALL patients require aggressive IV hydration during early treatment and are at risk of tumor lysis syndrome (TLS), with most studies noting an incidence of 20 to $45 \% .^{21,22}$ TLS increases the risk of AKI and presence of hyperuricemia at diagnosis is an independent risk factor for HTN development during induction chemotherapy. ${ }^{6}$ Patients with ALL are also at increased risk of sepsis, which greatly increases risk of AKI. ${ }^{23,24}$ Renal leukemic infiltration has also been identified as a risk factor in HTN development for these patients; however, in one study only $15.6 \%$ of patients with renal leukemic infiltration suffered from HTN, suggesting that renal leukemic infiltration alone is not causative. ${ }^{6}$ Nevertheless, these are some of the more significant factors that suggest that the leukemic process itself may be an important contributing etiologic factor.

\section{Consequences of Hypertension}

Hypertension causes accelerated vascular aging as well as a variety of other unwanted long-term sequelae leading to end-organ damage. ${ }^{4}$ HTN has also been found to be the most significant risk factor for development of posterior reversible encephalopathy syndrome (PRES) during pediatric cancer treatment. ${ }^{25}$ Additionally, HTN increases the risk for the development of renal parenchymal disorders and symptomatic osteonecrosis. ${ }^{6,26}$ Many previous studies have also shown that HTN during childhood is an independent risk factor for HTN as an adult. ${ }^{3,4}$

\section{Risks for ALL Survivors}

Children undergoing treatment for ALL are exposed to chemotherapy and radiotherapy during a critical period of their physiologic development and are vulnerable to side effects and complications from cancer treatment, increasing their risk of long-term effects, both morbidity and mortality. Many previous studies have demonstrated that childhood ALL survivors are at an increased risk of cardiovascular disease including HTN, heart failure, coronary artery disease, atherosclerosis, cardiac arrest, and stroke compared with the general population. ${ }^{27-30}$ The prevalence of HTN in childhood ALL survivors may be as high as $37 \%$, or 2.5 times that of the general population. ${ }^{30}$ In addition to the risk of cardiovascular disease, $12-39 \%$ of childhood ALL survivors develop features of metabolic syndrome including obesity, insulin resistance, and dyslipidemia. ${ }^{11,31-33}$ Childhood cancer survivors have been found to be about 8 times more likely to die due to cardiac-related events compared to the general population. ${ }^{34}$ Additionally, one large study found that HTN was a significant risk factor for the occurrence of potentially fatal cardiac events in survivors, independent of cancer therapy-related risk. ${ }^{35}$ Although some changes in therapy have been made over time, as discussed above with lesser cranial radiation, many of these factors for risk of HTN in childhood ALL survivors remain.

It has been well described that the rate of HTN in childhood ALL survivors is significantly higher than that of the healthy population; however, the mechanism for this is not fully understood. The toxic effects of chemotherapy on the developing cardiovascular system are known to cause damage to endocrine organs affecting hormones throughout the body, afflict the vascular endothelium affecting its function, and alter adipose tissue metabolism. ${ }^{36}$

\section{Challenges to Recognition and Management of Hypertension in the Hospital}

Children with new diagnoses of ALL typically start their first cycle of chemotherapy, known as induction or reduction chemotherapy, while admitted to the hospital. However, while pediatric HTN has been well studied in the ambulatory setting, with the 2017 AAP guidelines based entirely on BP readings of healthy children in 
a highly controlled ambulatory setting, our understanding of elevated BPs and HTN in hospitalized children remains limited. ${ }^{37}$ Many challenges exist in the inpatient setting for recognizing and managing HTN in a manner similar to that in the outpatient setting, as with ambulatory BP monitoring. ${ }^{38}$ First and foremost, accurate blood pressure monitoring in the inpatient setting can be practically quite difficult. Imprecise blood pressure measurements are frequently observed in the hospitalized pediatric population and are affected by physiologic conditions unique to the inpatient setting that cause transient elevations in blood pressure. Several issues contributing to this include the young age of this population, patients' agitation, anxiety, or pain, location of BP measurement, improper use of automatic BP machines, and concomitant medications. For many reasons, elevated BP recordings may go unrecognized or may be readily attributed to these confounding factors in the inpatient setting. It is imperative for providers to carefully analyze BP readings of hospitalized children to determine if elevations are due to a treatable cause such as pain or anxiety or are an indication of true HTN. ${ }^{37}$

A second significant challenge is the increasing evidence, primarily in adult literature but also in pediatric literature, that treatment of inpatient hypertension, or intensification of an antihypertensive regimen in the inpatient setting, does not always improve outcomes, including improved BP control outpatient, subsequent AKI, myocardial injury, or stroke. ${ }^{39}$ For this reason, we present for consideration the recommended treatment algorithm below for severe or persistently sustained HTN while attempting to avoid treating transient, non-sustained HTN. In this manner, HTN which is more likely to lead to long-term compromise can be managed more rapidly, while reducing the risk of treating HTN that is less likely to cause on-going or escalating physiologic damage and consequences.

While hypertensive emergencies are uncommon in this population, it is critical that they are recognized quickly and treated appropriately. More commonly in this population and in the hospital setting, persistent mild to moderate blood pressure elevations are observed. Appropriate management depends upon the correct clinical distinction of not only hypertensive emergencies, but also hypertensive urgencies versus persistent HTN. The clinical practice guidelines are helpful by providing relative cut-offs for hypertensive definitions, but clinical judgment must always play a role in distinguishing persistent HTN from the characteristics of a hypertensive crisis, including identifying signs of end organ damage in the case of emergencies. In the inpatient setting, unless patients have longstanding HTN, which is unlikely to resolve in the short term (ie, over a period of days), treatment decisions should revolve around the prevention of complications of acute, symptomatic HTN and the prevention of chronic renovascular disease. ${ }^{37}$

\section{Inpatient Approach to Hypertension in Pediatric ALL Patients}

In the setting of an elevated BP reading in the hypertensive range of $\geq 95$ th percentile or $\geq 130 / 80$ in those 13 years or older, first obtain a repeat blood pressure taken manually, in an upper extremity, ensuring appropriate cuff size for patient, and when patient is calm. Evaluate for possible medical causes of elevated BP including pain, agitation, anxiety, increased ICP, volume overload, or medications. Medications known to cause elevated BP include corticosteroids, stimulants, sympathomimetics (including decongestants), MAOIs, some OCPs, as well as abrupt discontinuation of clonidine or beta blockers. If an identifiable cause for elevated BP is suspected, treatment for that cause is indicated and repeat blood pressures should be obtained after appropriate treatment. If repeat BP is $<95$ th percentile $+12 \mathrm{mmHg}$ or $<140 / 90$ (whichever is lower), continue to monitor per institutional standards and good clinical judgement. If BP remains elevated on repeat manual BP measurement of $>95^{\text {th }}$ percentile $+12 \mathrm{mmHg}$ or $>140 / 90$, continue down the inpatient algorithm (Figure 1). If a patient has symptomatic HTN including altered mental status, chest pain, dyspnea, EKG changes, papilledema, or any other evidence of end organ damage, manage the patient as an hypertensive emergency, consult nephrology colleagues, and consider transfer to higher level care/ICU for closer monitoring and intervention.

If patient meets criteria for HTN but is asymptomatic or has only mild, non-specific symptoms (like mild headache or nausea), proceed down the left-hand side of the inpatient algorithm. Assess for AKI with labs (ie, basic metabolic panel including phosphorus), urinalysis, and echocardiogram (if patient does not have a recent echocardiogram). The patient's fluid balance should be monitored with strict intake and output measurements, along with daily weights. If any of these assessments are abnormal, nephrology consult (with or without cardiology consult, depending on individual institutional standards) and 
renal ultrasound may be indicated. Simultaneously, while lab and imaging workups are underway, evaluate for the presence of hypertensive risk factors inpatient including corticosteroid use, family history of HTN, medications known to elevate BP, any history of HTN for patient, history of AKI, prematurity, or known renal anomalies. If the patient has evidence of volume overload as a possible cause for HTN, treat with IV diuretics such as furosemide or bumetanide, and monitor BP following treatment. If no evidence of volume overload exists in the patient, intermittent as-needed medications can be administered to lower BP, based on severity of BP elevation or known risk factors for HTN (see Figure 1). If the patient continues to require PRN medications for elevated BP after 12 days, consult nephrology (if not previously consulted) and start a maintenance antihypertensive, such as amlodipine, if HTN is persistent.

Amlodipine, a calcium channel blocker that is a vasodilator used to improve blood flow, is often the longacting medication of choice as it is well tolerated and has been well studied with a favorable side effect profile in children (no increased risk of AKI). The primary side effects include edema often in the lower extremities and gingival hypertrophy, both of which are dose dependent and typically reversible within 2-3 months of cessation of the medication. Diuretics, such as the thiazide diuretics, may also be helpful, especially in the setting of steroid therapy or if volume retention has been an issue. Angiotensin-converting enzyme (ACE) inhibitor and angiotensin receptor blocker therapy is typically a first line in pediatric HTN but often avoided in the inpatient setting due to risk of AKI, especially when used in patients who are receiving concomitant or multiple nephrotoxic agents. However, these medications can be very effective and can be successfully used with close monitoring for AKI and in patients without notable risk factors for AKI. Application of a clonidine patch or scheduled oral clonidine can be an alternative, though not usually as first line intervention. Additionally, while beta blockers are alternatives, they are typically not as well tolerated in the pediatric population.

After starting long-acting oral medication for BP control, after 2-3 days of consistent dosing or steady state based on half-life of the agent, assess whether BP is improved to $\leq 95^{\text {th }}$ percentile or $<140 / 90$. If BP has responded appropriately to medication by meeting these criteria, continue the current dose of the medication. If BP remains elevated after 2-3 days of consistent dosing/steady state of the medication, increase the dose and reassess BP after 2-3 days/steady state at the new dose. If BP responds to the higher dose of medication, continue treating at this dose. If $\mathrm{BP}$ has not responded after increasing dose, nephrology consult is indicated at this time for assistance with further management of HTN.

\section{Outpatient Follow-Up}

All patients with childhood ALL should have blood pressures measured at all visits. Those who had been referred to nephrology or cardiology consultants during admission should continue to follow-up with the relevant consultants as outpatients in conjunction with their prescribed oncology care. Patients who develop or continue to have blood pressures in the hypertensive range $\left(\geq 95^{\text {th }}\right.$ percentile or $\geq 130 / 80$ for adolescents), should be referred for further evaluation and 24-hour ambulatory blood pressure monitoring (ABPM). Likewise, those who are on treatment but remain in the hypertensive range should be referred for ABPM. When the HTN resolves, children $\geq 6$ years old on amlodipine with doses of $5 \mathrm{mg}$ or less may discontinue and if the dose is $>5 \mathrm{mg}$ per day, decrease by half the dose and then stop after 2 weeks if remaining normotensive. Likewise, for younger children, if dose is greater than the starting dose, decrease the dose by half and then if they remain normotensive, discontinue after two weeks. For thiazide diuretics, if at the recommended starting dose for age, patients can discontinue and monitor for weight gain and/or increased BP. If treated above the starting dose for age, consider decreasing the dose by half and then stopping after 1-2 weeks if patients remain normotensive. If blood pressure increases to $>95^{\text {th }}$ percentile for age during weaning or after discontinuation, referral for evaluation and a 24-hour ABPM is most appropriate. Please see Figure 2 for an algorithmic approach.

Patients with other identified risk factors for HTN, such as other cardiac disease or obesity, in addition to ALL and steroid treatment are those that are most likely to continue to require antihypertensive medication after ALL treatment. Those patients who had AKI during their treatment may require antihypertensive therapy longer, with possible discontinuation, as compared to those who did not have AKI. All patients with sustained HTN are at increased risk for recurrence of HTN as compared to their counterparts without sustained HTN. Thus, patients with ALL and especially those who required treatment for HTN, should be counseled for the need for at least annual 


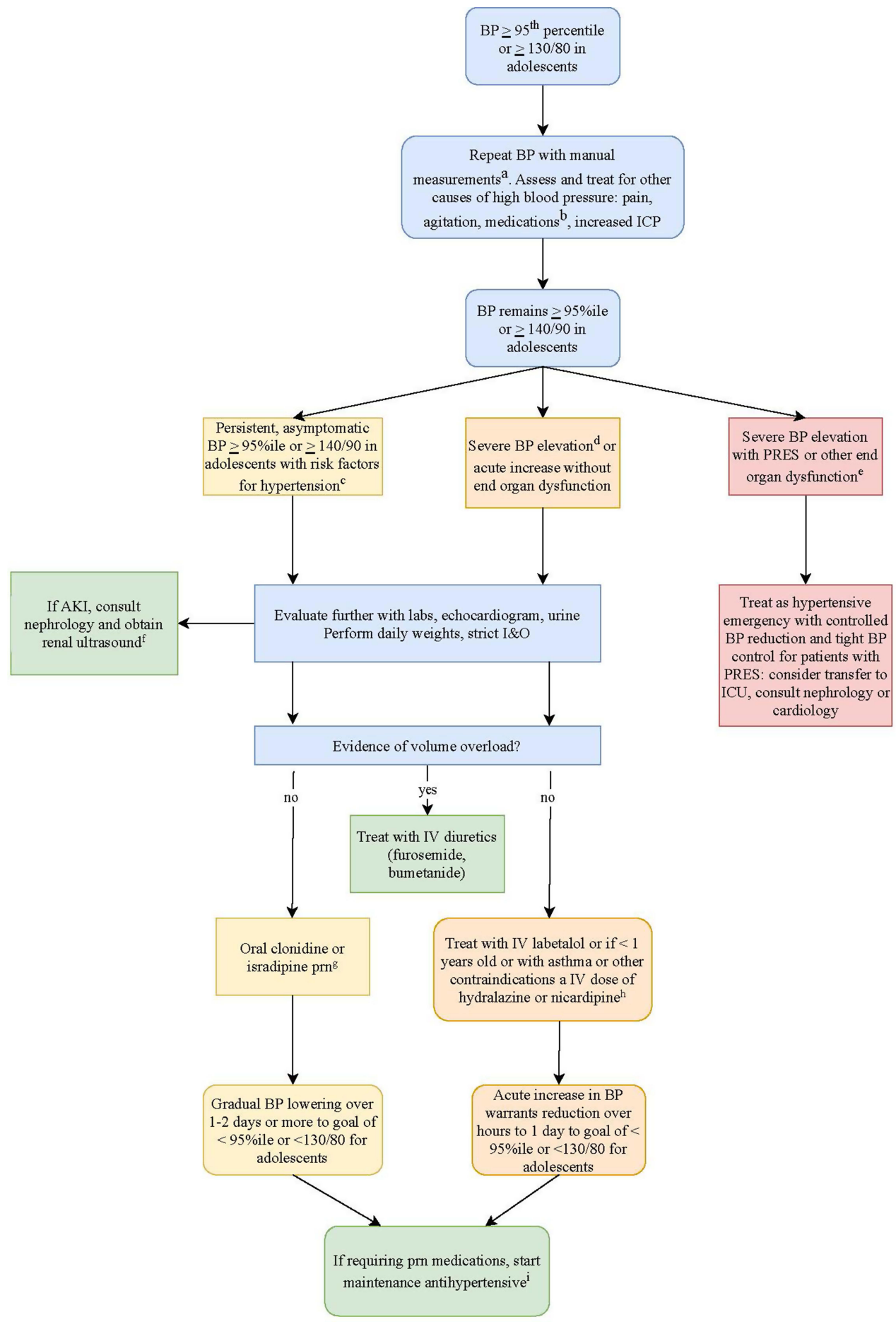

Figure I Inpatient hypertension algorithm pediatric patients with acute lymphoblastic leukemia. ${ }^{a}$ Ensure appropriately sized cuff, taken in upper extremity when patient is resting and calm. bMedications that can cause hypertension include corticosteroids, stimulants, sympathomimetics (decongestants), MAOls, some OCPs, and abrupt discontinuation of clonidine or beta blockers. 'Common risk factors in addition to corticosteroid exposure include prematurity, family history of hypertension, past history of acute kidney injury, chronic kidney disease, coarctation of the aorta, certain genetic syndromes. ${ }^{\mathrm{d}}$ Severe elevation is typically defined as $>30 \mathrm{mmHg}$ above the 95 th percentile for age, height, and sex or > 170/1 10; however, there is no precise measurement cut-off due to presence or risk of end organ dysfunction. ${ }^{\mathrm{e}} \mathrm{Hypertensive}$ encephalopathy including seizures and manifestations of PRES, AKI due to hypertension, heart failure. 'Recommend renal ultrasound without Doppler as sensitivity for renovascular hypertension is limited with Doppler and a CT angiogram or MR angiogram are recommended for high suspicion of renovascular hypertension. ${ }^{8} \mathrm{Clonidine}$ initial starting dose: $2-5 \mathrm{mcg} / \mathrm{kg} /$ dose every 6-8 hours as needed, up to $10 . \mathrm{mcg} / \mathrm{kg} /$ dose. Isradipine initial starting dose: $0.05-0.1 \mathrm{mg} / \mathrm{kg} /$ dose every 6-8 hours as needed, maximum starting dose of $2.5 \mathrm{mg}$ per dose. "Labetalol initial starting dose: $0.2-1 \mathrm{mg} / \mathrm{kg} /$ dose every $4-6$ hours as needed, up to $40 \mathrm{mg}$ per dose. IV Hydralazine initial starting dose: $0.1-$ $0.2 \mathrm{mg} / \mathrm{kg} / \mathrm{dose}$ every 4-6 hours as needed, max of $20 \mathrm{mg}$ per dose. IV Nicardipine initial starting dose: $30 \mathrm{mcg} / \mathrm{kg} / \mathrm{dose}$, maximum of $2 \mathrm{mg}$ per dose as optional bolus prior to starting IV infusion in ICU. 'Amlodipine initial starting dose for children $<6$ years: $0.1 \mathrm{mg} / \mathrm{kg} /$ day divided I-2 times per day, For children $>6$ years, initial dose of $2.5 \mathrm{mg}$ once daily; Ace-inhibitors can also be considered with starting dose of lisinopril $0.07 \mathrm{mg} / \mathrm{kg} /$ day once daily, with maximum initial dose of $5 \mathrm{mg} / \mathrm{day}$.

Abbreviations: BP, blood pressure; AKI, acute kidney injury; PRES, posterior reversible encephalopathy syndrome. 


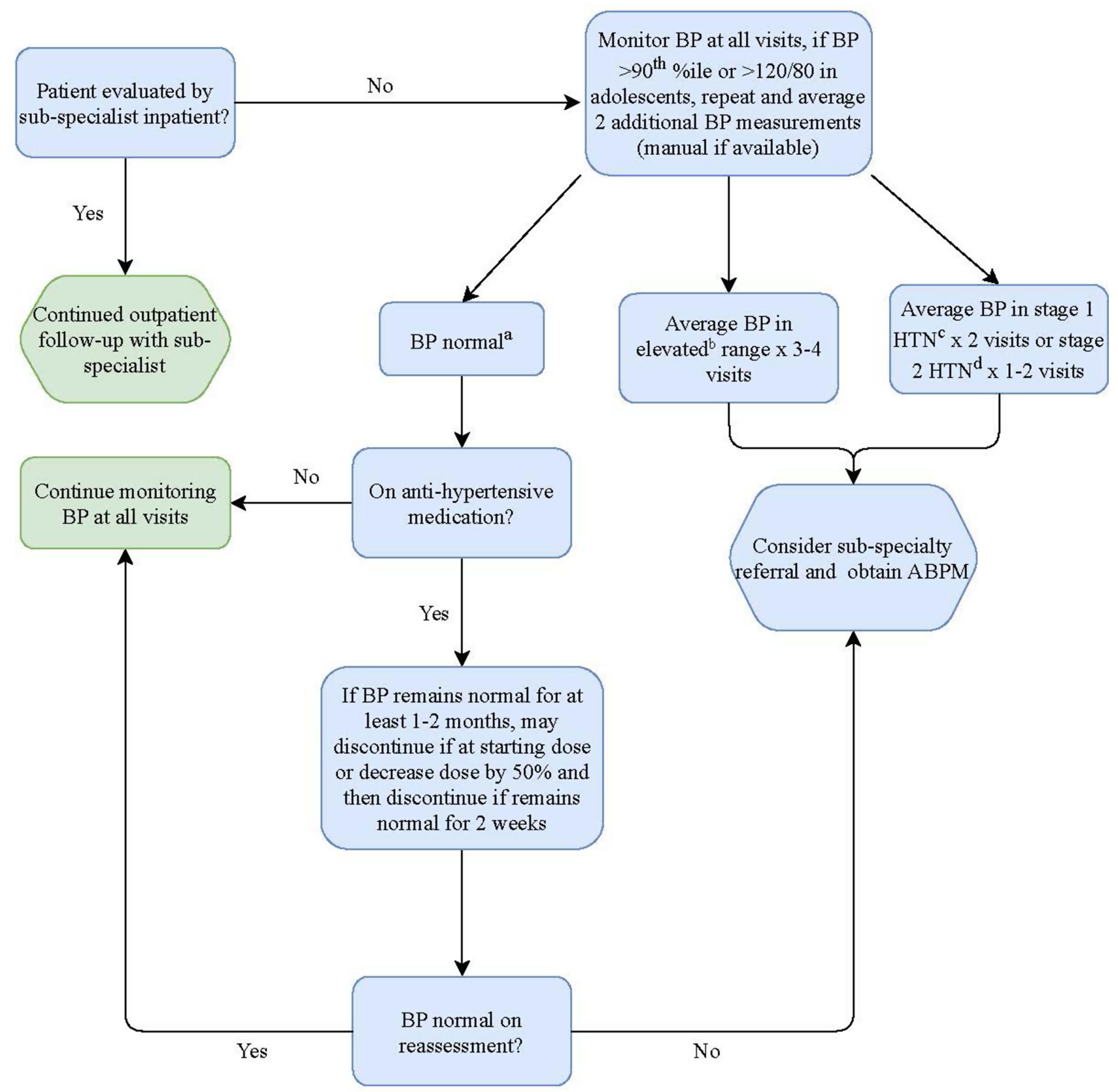

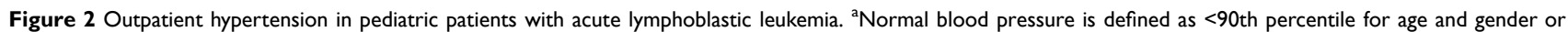
$<120 / 80$ for adolescents. ${ }^{b} E$ Elevated blood pressure is defined as $\geq 90$ th percentile for age and gender and $<95$ th percentile or $\geq 120 / 80$ and $<130 / 80$ for adolescents. ${ }^{c}$ Stage $I$ hypertension is defined as $\geq 95$ th percentile for age and gender and $<95$ th percentile $+15 \mathrm{mmHg}$ or $\geq 130 / 80$ and $<140 / 90$ for adolescents. ${ }^{\mathrm{d}}$ Stage 2 hypertension is defined as $\geq 95$ th percentile $+15 \mathrm{mmHg}$ or $\geq 140 / 90$ for adolescents. The definitions are based on those from the 2017 clinical practice guideline for screening and management of high blood pressure in children and adolescents.

Abbreviations: BP, blood pressure; HTN, hypertension; ABPM, 24-hour ambulatory blood pressure monitor.

visits with blood pressure assessment and follow-up of any elevated blood pressures.

\section{Discussion}

Hypertension is a well-known but under-recognized complication in children undergoing treatment for pediatric ALL. As described, the rates of HTN in pediatric patients, especially during the first (induction or reduction) phase of therapy are significant. Many pediatric patients with ALL require antihypertensive medications during some portion of their treatment. However, clear guidelines on treating inpatient HTN in this population are lacking as well as on monitoring and continuation versus cessation of anti-HTN therapy as an outpatient. 
Most importantly in this population, while elevated BPs may be common, if not recognized and treated promptly, significant consequences can result. Treatment recommendations for children with HTN are based on the goal of preventing long-term systemic complications. This goal should be the same for pediatric ALL patients. However, for most children, elevated blood pressures are subacute and resolve before the end of therapy. While longstanding HTN is unlikely, especially if diagnosed in the inpatient setting, clinical detection of hypertensive urgencies and emergencies is of utmost importance to prevent acute, symptomatic HTN complications as well.

Hypertension is a common acute and late complication of pediatric leukemia treatment. Additionally, patients who were treated for HTN previously have increased long-term risk of recurrence of HTN and immediate assessment and intervention may be indicated. These patients should have long-term monitoring for recurrence of HTN.

A standardized approach for accurately diagnosing and treating HTN in pediatric ALL patients has not existed consistently and is an unmet medical need for children with ALL. While the HTN guidelines for pediatrics as a whole can be helpful, patients with childhood ALL represent a unique population with several key factors to consider when diagnosing HTN. These include the confounding factors of accurate blood pressure monitoring in the inpatient setting, as well as the complicating aspects of leukemia treatment that predispose these children to blood pressure elevations, including steroids. We must recognize in this population that the fixed cut-offs proposed in the HTN guidelines are important to apply as a foundation for diagnosis, but that individual patient characteristics and other factors are critical to incorporate in the medical decision-making process.

Herein we propose an algorithmic approach to diagnose and treat pediatric ALL patients with HTN. Applying this standardized methodology for HTN in this population will allow for further future prospective and longitudinal studies to better assess the long-term outcomes and results of adequate treatment. In addition to the management suggestions proposed here, close long-term outpatient follow-up is needed for further monitoring of blood pressures along with titration or discontinuation of antihypertensive medication when applicable. The ultimate goal of treatment of childhood ALL is to cure patients and help them to have high-quality survivorship and healthy adult lives. Vigilant blood pressure management during therapy and careful follow-up care are essential components to ultimately achieving the best care for patients.

\section{Disclosure}

Dr Lia Gore reports an unpaid advisory board position from Amgen, Roche, and Janssen; advisory board position from Kura and OnKure; and stock from Mirati, outside the submitted work. The authors report no other conflicts of interest in this work.

\section{References}

1. Pui CH, Mullighan CG, Evans WE, Relling MV. Pediatric acute lymphoblastic leukemia: where are we going and how do we get there? Blood. 2012;120(6):1165-1174.

2. Silverman LB, Gelber RD, Dalton VK, et al. Improved outcome for children with acute lymphoblastic leukemia: results of Dana-Farber Consortium Protocol 91-01. Blood. 2001;97(5):1211-1218.

3. Meenan CK, Kelly JA, Wang L, Ritchey AK, Maurer SH. Obesity in pediatric patients with acute lymphoblastic leukemia increases the risk of adverse events during pre-maintenance chemotherapy. Pediatr Blood Cancer. 2019;66(2):e27515.

4. Flynn JT, Kaelber DC, Baker-Smith CM, et al. Clinical practice guideline for screening and management of high blood pressure in children and adolescents. Pediatrics. 2017;140:3.

5. National High Blood Pressure Education Program Working Group on High Blood Pressure in C, Adolescents. The fourth report on the diagnosis, evaluation, and treatment of high blood pressure in children and adolescents. Pediatrics. 2004;114(2 Suppl 4th Repor t):555-576.

6. Olgar S, Yetgin S, Cetin M, Aras T. Can renal leukemic infiltration cause hypertension in children? J Pediatr Hematol Oncol. 2006;28 (9):579-584.

7. Attard-Montalto SP, Saha V, Ng YY, Kingston JE, Eden OB. High incidence of hypertension in children presenting with acute lymphoblastic leukemia. Pediatr Hematol Oncol. 1994;11(5):519-525.

8. Kamdem LK, Hamilton L, Cheng C, et al. Genetic predictors of glucocorticoid-induced hypertension in children with acute lymphoblastic leukemia. Pharmacogenet Genomics. 2008;18(6):507-514.

9. Bakk I, Koch T, Stanek J, O’Brien SH, Reed S. Steroid-induced hypertension during induction chemotherapy for acute lymphoblastic leukemia in US Children's hospitals. J Pediatr Hematol Oncol. 2018;40(1):27-30.

10. Malhotra P, Kapoor G, Jain S, Garg B. Incidence and risk factors for hypertension during childhood acute lymphoblastic leukemia therapy. Indian Pediatr. 2018;55(10):877-879.

11. Abu-Ouf NM, Jan MM. Metabolic syndrome in the survivors of childhood acute lymphoblastic leukaemia. Obes Res Clin Pract. 2015;9(2):114-124.

12. Maloney KW, Devidas M, Wang C, et al. Outcome in children with standard-risk B-cell acute lymphoblastic leukemia: results of children's oncology group trial AALL0331. J Clin Oncol. 2020;38 (6):602-612.

13. Larsen EC, Devidas M, Chen S, et al. Dexamethasone and High-dose methotrexate improve outcome for children and young adults with highrisk B-acute lymphoblastic leukemia: a report from children's oncology group study AALL0232. J Clin Oncol. 2016;34(20):2380-2388.

14. Wirtz PH, von Kanel R, Emini L, et al. Evidence for altered hypothalamus-pituitary-adrenal axis functioning in systemic hypertension: blunted cortisol response to awakening and lower negative feedback sensitivity. Psychoneuroendocrinology. 2007;32(5):430-436.

15. Igarashi R, Fujihara K, Heianza Y, et al. Impact of individual components and their combinations within a family history of hypertension on the incidence of hypertension: Toranomon hospital health management center study 22. Medicine (Baltimore). 2016;95(38): e4564. 
16. Sorof J, Daniels S. Obesity hypertension in children: a problem of epidemic proportions. Hypertension. 2002;40(4):441-447.

17. Esbenshade AJ, Simmons JH, Koyama T, Koehler E, Whitlock JA, Friedman DL. Body mass index and blood pressure changes over the course of treatment of pediatric acute lymphoblastic leukemia. Pediatr Blood Cancer. 2011;56(3):372-378.

18. Agarwal M, Thareja N, Benjamin M, Akhondi A, Mitchell GD. Tyrosine Kinase Inhibitor-Induced Hypertension. Curr Oncol Rep. 2018;20(8):65

19. Armstrong GT, Chen Y, Yasui Y, et al. Reduction in late mortality among 5-year survivors of childhood cancer. N Engl J Med. 2016;374 (9):833-842.

20. Benisty K, Morgan C, Hessey E, et al. Kidney and blood pressure abnormalities 6 years after acute kidney injury in critically ill children: a prospective cohort study. Pediatr Res. 2020;88(2):271-278.

21. Tasmeen R, Islam A, Alam ST, Begum M. Tumor lysis syndrome and hyperleukocytosis in childhood acute lymphoblastic leukemia in a tertiary care hospital. Mymensingh Med J. 2017;26(4):906-912.

22. Belay Y, Yirdaw K, Enawgaw B. Tumor lysis syndrome in patients with hematological malignancies. J Oncol. 2017;2017:9684909.

23. Zhu Y, Yang R, Cai J, et al. Septicemia after chemotherapy for childhood acute lymphoblastic leukemia in China: a multicenter study CCCG-ALL-2015. Cancer Med. 2020;9(6):2113-2121.

24. Fitzgerald JC, Ross ME, Thomas NJ, Weiss SL, Balamuth F, Anderson AH. Risk factors and inpatient outcomes associated with acute kidney injury at pediatric severe sepsis presentation. Pediatr Nephrol. 2018;33(10):1781-1790.

25. de Laat P, Te Winkel ML, Devos AS, Catsman-Berrevoets CE, Pieters R, van den Heuvel-eibrink MM. Posterior reversible encephalopathy syndrome in childhood cancer. Ann Oncol. 2011;22 (2):472-478

26. Janke LJ, Van Driest SL, Portera MV, et al. Hypertension is a modifiable risk factor for osteonecrosis in acute lymphoblastic leukemia. Blood. 2019;134(12):983-986.

27. Haddy TB, Mosher RB, Reaman GH. Hypertension and prehypertension in long-term survivors of childhood and adolescent cancer. Pediatr Blood Cancer. 2007;49(1):79-83.

28. Levy E, Samoilenko M, Morel S, et al. Cardiometabolic risk factors in childhood, adolescent and young adult survivors of acute lymphoblastic leukemia - a petale cohort. Sci Rep. 2017;7(1):17684.

29. Chow EJ, Pihoker C, Hunt K, Wilkinson K, Friedman DL. Obesity and hypertension among children after treatment for acute lymphoblastic leukemia. Cancer. 2007;110(10):2313-2320.
30. Ociepa T, Bartnik M, Zielezinska K, Urasinski T. Prevalence and risk factors for arterial hypertension development in childhood acute lymphoblastic leukemia survivors. $J$ Pediatr Hematol Oncol. 2019;41(3):175-180.

31. England J, Drouin S, Beaulieu P, et al. Genomic determinants of long-term cardiometabolic complications in childhood acute lymphoblastic leukemia survivors. BMC Cancer. 2017;17(1):751.

32. Nottage KA, Ness KK, Li C, Srivastava D, Robison LL, Hudson MM. Metabolic syndrome and cardiovascular risk among long-term survivors of acute lymphoblastic leukaemia - From the St. Jude Lifetime Cohort. Br J Haematol. 2014;165(3):364-374.

33. Oeffinger KC, Buchanan GR, Eshelman DA, et al. Cardiovascular risk factors in young adult survivors of childhood acute lymphoblastic leukemia. J Pediatr Hematol Oncol. 2001;23(7):424-430.

34. Mertens AC, Yasui Y, Neglia JP, et al. Late mortality experience in five-year survivors of childhood and adolescent cancer: the Childhood Cancer Survivor Study. J Clin Oncol. 2001;19 (13):3163-3172.

35. Armstrong GT, Oeffinger KC, Chen Y, et al. Modifiable risk factors and major cardiac events among adult survivors of childhood cancer. $J$ Clin Oncol. 2013;31(29):3673-3680.

36. Ociepa T, Bartnik M, Zielezinska K, Prokowska M, Urasinska E, Urasinski T. Abnormal correlation of circulating endothelial progenitor cells and endothelin-1 concentration may contribute to the development of arterial hypertension in childhood acute lymphoblastic leukemia survivors. Hypertens Res. 2016;39(7):530-535.

37. Chaudhuri A, Sutherland SM. Evaluation and management of elevated blood pressures in hospitalized children. Pediatr Nephrol. 2019;34(10):1671-1681.

38. Hamdani G, Flynn JT, Daniels S, et al. Ambulatory blood pressure monitoring tolerability and blood pressure status in adolescents: the SHIP AHOY study. Blood Press Monit. 2019;24(1):12-17.

39. Rastogi R, Sheehan MM, Hu B, Shaker V, Kojima L, Rothberg MB. Treatment and outcomes of inpatient hypertension among adults with noncardiac admissions. JAMA Intern Med. 2021;181(3):345-352.

40. Falkner B, Daniels SR. Summary of the fourth report on the diagnosis, evaluation, and treatment of high blood pressure in children and adolescents. Hypertension. 2004;44(4):387-388.

41. NIH National Cancer Institute [webpage on the internet]. Cancer Therapy Evaluation Program (CTEP). [Updated September 21, 2020].. Available from .https://ctep.cancer.gov/protocoldevelopment/ electronic_applications/Ctc.htm. Accessed January 11, 2022.
Integrated Blood Pressure Control

\section{Publish your work in this journal}

Integrated Blood Pressure Control is an international, peer-reviewed open-access journal focusing on the integrated approach to managing hypertension and risk reduction. Treating the patient and comorbidities together with diet and lifestyle modification and optimizing healthcare resources through a multidisciplinary team approach constitute key features of the journal. This journal is indexed on
American Chemical Society’s Chemical Abstracts Service (CAS). The manuscript management system is completely online and includes a very quick and fair peer-review system, which is all easy to use. Visit http://www.dovepress.com/testimonials.php to read real quotes from published authors. 